\title{
The Bridge Tournament Problem and Calibration Designs for Comparing Pairs of Objects
}

\author{
R. C. Bose* and J. M. Cameron
}

(August 17, 1965)

\begin{abstract}
The classical tournament problem calls for arranging $v$ individuals into teams of $p$ players so that a player is teamed the same number of times with each of the other players and also that each player is pitted equally often against each of the other players. The play of the tournament results in the determination of difference in performance of the various pairings of the groups. In the special case when $p=2$ each team consists of two players and the designs are called bridge tournament designs.

In high precision calibration one can measure only the difference between two nominally equal groups so that if $v$ objects are to be intercompared in groups of $p$ objects, then the solutions to the tournament problem provide schedules for the grouping. These designs are useful in weighing and any other measurements where the objects to be measured can be combined into groups without loss of precision or accuracy in the comparisons.

This paper presents general methods for constructing of bridge tournament designs, i.e., for the case when $p=2$, for all $v \leqslant 50$.
\end{abstract}

Key Words: Calibration, calibration designs, combinatorial analysis, difference sets, experiment designs, incomplete block designs, tournaments, weighing designs.

\section{Introduction}

The classical tournament problem calls for arranging $v$ individuals into teams of $p$ players, so that each player is teamed the same number of times with each of the other players, and also each player is pitted equally often against each of the other players. Consistent with these conditions one would like to minimize the number of matches. Such an arrangement may be called a tournament design. Each match corresponds to a block of this design, the block being divided into two half-blocks corresponding to the two teams. When the tournament is played according to this design, it facilitates the determination of the difference in performance between the various pairs of players.

In high precision calibration one can measure the difference between two nominally equal groups so that if $v$ objects are intercompared in groups of $p$ objects then tournament designs provide schedules for grouping into sets. These designs are useful in weighing and other measurements where the objects to be measured can be combined into groups without loss of precision or accuracy in the comparisons.

In the special case when $p=2$, each team consists of two players, which is the situation in a bridge match. The corresponding designs can then be called bridge tournament designs. When used for calibration, two pair of objects are compared with each other.

In this paper we give general methods for constructing bridge tournament designs, and obtain in particular all designs for $v \leqq 50$. The statistical analysis for the corresponding calibration designs will appear elsewhere. The tournament designs for $p>2$ will be considered in a separate communication. For previous work on the same problem, reference should be made to $\left[7,{ }^{1} 10,13\right.$, and 14$]$.

\section{Parameters for Tournament Designs}

Let there be $v$ players or objects. We have to arrange them in $b$ blocks of size $2 p$, each block consisting of two half-blocks of size $p$. Two objects appear in the same half block $\lambda_{1}$ times, and in opposite half-blocks of the same block $\lambda_{2}$ times. Then

$$
v, b, r, p, \lambda_{1}, \lambda_{2},
$$

are said to be the parameters of the tournament design, where $r$ is the number of blocks in which each object appears.

Consider the $r$ blocks in which a given object $\theta$ appears. Then there are $r(p-1)$ other objects in the half-blocks containing $\theta$. Since each of the $v-1$ objects other than $\theta$, appears $\lambda_{1}$ times in the same halfblock as $\theta$,

$$
\lambda_{1}(v-1)=r(p-1) .
$$

Similarly

$$
\lambda_{2}(v-1)=r p
$$


Hence $r=\left(\lambda_{2}-\lambda_{1}\right)(v-1)$, which shows that $r$ must be divisible by $v-1$. We can take $r=\beta(v-1)$. Let there be $b$ blocks (or matches). Then counting the number of objects in the $b$ blocks in two different ways we have

$$
2 p b=v r=v(v-1) \beta .
$$

Hence

$$
b=\beta v(v-1) / 2 p .
$$

Let $h$ be the highest common factor of $v(v-1)$ and $2 p$ and let $2 p=h n$. Then $\beta=\lambda_{2}-\lambda_{1}$ must be divisible by $n$, hence the least possible value of $\beta$ is $n$. If the corresponding design exists we shall call it minimal in the sense that no smaller number of blocks could possibly lead to a balanced design, i.e., integer values for $\lambda_{1}$ and $\lambda_{2}$.

The parameters of a minimal design are

$$
\begin{aligned}
v, b=v(v-1) / h, r=n(v-1), & p, \\
\lambda_{1} & =n(p-1), \lambda_{2}=n p,
\end{aligned}
$$

where $h$ is the highest common factor of $v(v-1)$ and $2 p=h n$.

Now consider the special case $p=2$. In this case $h=4, n=1$, if $v=4 t+1$ or $4 t$, and $h=2, n=2$ if $v=4 t+3$ or $4 t+2$. Hence we have the following four series of minimal bridge tournament designs.

$$
\text { (A) } \begin{aligned}
v=4 t+3, b=(4 t+3)(2 t+1), \\
r=2(4 t+2), p=2, \lambda_{1}=2, \lambda_{2}=4,
\end{aligned}
$$

(B) $v=4 t+1, b=t(4 t+1), r=4 t$,

$$
p=2, \lambda_{1}=1, \lambda_{2}=2,
$$

(C) $v=4 t+2, b=(2 t+1)(4 t+1)$,

$$
r=2(4 t+1), p=2, \lambda_{1}=2, \lambda_{2}=4,
$$

(D) $v=4 t, b=t(4 t-1), r=4 t-1$.

$$
p=2, \lambda_{1}=1, \lambda_{2}=2 \text {. }
$$

We shall show that an infinity of designs exist for each series. In particular all the designs for $v \leqq 50$ exist, and will be explicitly obtained.

\section{The Method of Symmetrically Repeated Differences}

Tournament designs are very closely related with balanced incomplete block (BIB) designs.

A BIB design is an arrangement of $v^{*}$ objects in $b^{*}$ blocks or sets such that (i) each block contains exactly $k^{*}$ different objects (ii) each object appears in exactly $r^{*}$ blocks (iii) any pair of distinct objects appear together in exactly $\lambda^{*}$ blocks. The BIB design is then said to have parameters $v^{*}, b^{*}, r^{*}, k^{*}, \lambda^{*}$. It is evident that the necessary and sufficient condition for the existence of a tournament design with parameters (2.1) is the existence of a BIB design with parameters

$$
v^{*}=v, b^{*}=b, r^{*}=r, k^{*}=2 p, \lambda^{*}=\lambda_{1}+\lambda_{2},
$$

each of whose blocks can be divided into two halfblocks such that the half-blocks form a BIB design with parameters

$$
v_{0}=v, b_{0}=2 b, r_{0}=r, k_{0}=p, \lambda_{0}=\lambda_{1} .
$$

The method of symmetrically repeated differences which was originally developed by Bose [1], in connection with the construction of BIB designs, can be adapted to the construction of tournament designs. An excellent exposition of the method will be found in Mann [11]. We shall give here the necessary definitions and quote the two basic theorems, without introducing the concepts of mixed differences which will not be explicitly needed in this paper.

Consider a module $M$ with $n$ elements. If $a_{1}$ and $a_{2}$ are any elements of $M$ we say that the elements $\pm\left(a_{1}-a_{2}\right)$ are the differences arising from the pair $a_{1}, a_{2}$. Let the objects be put in one-to-one correspondence with the elements of $M$. Given a block $B$ with $m$ elements $a_{1}, a_{2}, \ldots, a_{m}$, the $m(m-1)$ differences $\pm\left(a_{i}-a_{j}\right), i, j=1,2, \ldots, m, i \neq j$, arising from all distinct pairs of objects in the block, are called the differences arising from the block. Given a set of blocks such that among the differences arising from the blocks of the set, each nonzero element of $M$ occurs exactly $\lambda$ times, we say that the differences arising from the set of blocks are symmetrically repeated $\lambda$ times. From the block $B=\left(a_{1}, a_{2}, \ldots, a_{m}\right)$ we can form a set of $n$ blocks $\left(a_{1}+\theta, a_{2}+\theta, \ldots, a_{m}+\theta\right)$, where $\theta$ runs over all the different elements of $M$. This set of $n$ blocks is said to be obtained by developing the initial block $B$. We can now enunciate the following theorem:

THEOREM 1. Let $\mathrm{M}$ be a module containing $\mathrm{v}^{*}$ elements, which have been put in one-to-one correspondence with a set of $\mathrm{v}^{*}$ objects. If there exists a set of $\mathrm{t}$ blocks

$$
\mathrm{B}_{1}, \mathrm{~B}_{2}, \ldots, \mathrm{B}_{\mathrm{t}}
$$

such that (i) Each block contains k* distinct objects (ii) the differences arising from the blocks are symmetrically repeated $\lambda^{*}$ times, then we get a BIB design with parameters

$$
\mathrm{v}^{*}, \mathrm{~b}^{*}=\mathrm{v}^{*} \mathrm{t}, \mathrm{r}^{*}=\mathrm{k}^{*} \mathrm{t}, \mathrm{k}^{*}, \lambda^{*}
$$

by developing the initial blocks (3.3).

THEOREM 2. Let $\mathrm{M}$ be a module containing $\mathrm{n}$ elements, which have been put in one-to-one correspondence with a set of $\mathrm{n}$ objects. To these adjoin a new object $\propto$. If it is possible to find a set of $\mathrm{g}+\mathrm{s}$ blocks

$$
\mathrm{B}_{1}, \mathrm{~B}_{2}, \ldots, \mathrm{B}_{\mathrm{g}}, \mathrm{B}_{1}^{\prime}, \mathrm{B}_{2}^{\prime}, \ldots, \mathrm{B}_{s}^{\prime} \text {; }
$$

such that (i) Each of the blocks $\mathrm{B}_{1}, \mathrm{~B}_{2}, \ldots$, , $\mathrm{B}_{\mathrm{g}}$ contains 
$\mathrm{k}^{*}$ distinct objects which are all different from $\propto$ while each of the blocks $\mathrm{B}_{1}^{\prime}, \mathrm{B}_{2}^{\prime}, \ldots . \mathrm{B}_{s}^{\prime}$ contains $\propto$ and $\mathrm{k}^{*}-1$ other distinct objects, (ii) The differences arising from the blocks $\mathrm{B}_{1}, \mathrm{~B}_{2}, \ldots \mathrm{B}_{\mathrm{t}}, \mathrm{B}_{1}^{\prime \prime}, \mathrm{B}_{2}^{\prime \prime}, \ldots, \mathrm{B}_{\mathrm{s}}^{\prime \prime}$, where the blocks $\mathrm{B}_{\mathrm{j}}^{\prime \prime}$ are obtained from $\mathrm{B}_{\mathrm{j}}^{\prime}(\mathrm{j}=1,2, \ldots \mathrm{s})$ by cut ting out the adjoined object $\propto$, are symmetrically repeated $\lambda^{*}$ times, (iii) $\mathrm{k}^{*} \mathrm{~g}+\lambda^{*}=\mathrm{ns}, \lambda^{*}=\left(\mathrm{k}^{*}-1\right) \mathrm{s}$ then we get a BIB design with parameters

$$
\mathrm{v}^{*}=\mathrm{n}+1, \mathrm{~b}^{*}=\mathrm{n}(\mathrm{g}+\mathrm{s}), \mathrm{r}^{*}=\mathrm{ns}, \mathrm{k}^{*}=\mathrm{k}, \lambda^{*}=\lambda,
$$

by developing the blocks (3.5), where $\propto$ remains unchanged during development.

For a proof of slightly more general theorems see Bose [1], pp. 366-372 and Mann [11], pp. 115-118.

\section{Designs Belonging to the Series When $4 t+3$ is a Prime Power}

In the remainder of this paper we shall consider only bridge tournament designs, i.e., the special case $p=2$ of general tournament designs. When the module $M$ is a commutative ring or a field it will be be convenient to use the notation

$$
\left\{\left(a_{1}, a_{2}\right),\left(b_{1}, b_{2}\right)\right\} \oplus\left(c_{1}, c_{2}, \ldots c_{u}\right),
$$

to denote the set of blocks

$$
\left\{\left(a_{1} c_{i}, a_{2} c_{i}\right),\left(b_{1} c_{i}, b_{2} c_{i}\right)\right\}, i=1,2, \ldots, u .
$$

If $4 t+3=q$ is a prime power, we can identify the $v$ objects or players with elements of the Galois field $G F(q)$. Let $x$ be a primitive element of $G F(q)$, and let $a_{1}, a_{2}, b_{1}, b_{2}$ be any four nonzero distinct elements of $G F(q)$.

Now $x^{4 t+2}=1$ and $x^{2 t+1}=-1$, so that -1 is an odd power of $x$. It follows that if $c$ and $d$ are any distinct elements of $G F(q)$ the differences arising from the pairs

$$
(c, d),\left(c x^{2}, d x^{2}\right), \ldots\left(c x^{4 t}, d x^{4 t}\right),
$$

are symmetrically repeated once.

Now consider the set of $2 t+1$ blocks

$$
\left\{\left(a_{1}, a_{2}\right),\left(b_{1}, b_{2}\right)\right\} \oplus\left(1, x^{2}, x^{4}, \ldots x^{4 t}\right) .
$$

If we take the $4 t+2$ half-blocks from (4.3) as initial blocks, the differences arising from these are symmetrically repeated twice. Hence we get a BIB design with parameters

$v_{0}=4 t+3, b_{0}=2(4 t+3)(2 t+1)$,

$$
r_{0}=4(2 t+1), k_{0}=2, \lambda_{0}=2 .
$$

Similarly if we take the $2 t+1$ blocks in (4.3) as initial blocks (without partitioning into half-blocks), we get a BIB design with parameters

$$
\begin{aligned}
& v^{*}=4 t+3, b^{*}=(4 t+3)(2 t+1), \\
& r^{*}=4(2 t+1), k^{*}=4, \lambda^{*}=6 .
\end{aligned}
$$

It follows that if we develop the initial blocks (4.3), keeping the half-blocks intact, we get a bridge tournament design belonging to the series A.

In particular we get the solutions for $v=7,11,19,23$, $27,31,43,47$. For $v \leqslant 50$ this leaves only the values 15,35 , and 39 of the form $4 t+3$. These will be con-

\begin{tabular}{|c|c|c|}
\hline $\begin{array}{llll}12 & 3 & 4\end{array}$ & $\begin{array}{llll}2 & 4 & 6 & 1\end{array}$ & 41 \\
\hline 2345 & $\begin{array}{llll}3 & 5 & 0 & 2\end{array}$ & 52 \\
\hline $\begin{array}{llll}3 & 4 & 5 & 6\end{array}$ & 46.13 & 63 \\
\hline $\begin{array}{lll}5 & 6 & 0\end{array}$ & $\begin{array}{llll}5 & 0 & 2 & 4\end{array}$ & 04 \\
\hline $\begin{array}{lll}6 & 0 & 1\end{array}$ & $\begin{array}{llll}6 & 1 & 3 & 5\end{array}$ & \\
\hline 12 & $\begin{array}{llll}0 & 2 & 4 & 6\end{array}$ & 26 \\
\hline 123 & $\begin{array}{llll}13 & 5 & 0\end{array}$ & 30 \\
\hline
\end{tabular}
sidered in section 14 .

ExAMPLE (1). Let $t=1$. The required design has the parameters $v=7, b=21, r=12, p=2, \lambda_{1}=2, \lambda_{2}=4$. Now 3 is a primitive element of $G S(7)$. Hence the design can be developed from the initial blocks

$$
\{(1,2),(3,4)\} \oplus(1,2,4)
$$

which leads to the design

ExAmple (2). Let $t=2$. The required design has parameters $v=11, b=55, r=20, p=2, \lambda_{1}=2, \lambda_{2}=4$. A primitive element of $G F(11)$ is 2 . The design can be developed from the initial blocks

$$
\{(1,2),(3,4)\} \oplus(1,4,5,9,3) .
$$

\section{Designs Belonging to the Series (B), When $4 t+1$ is a Prime Power}

Let $4 t+1=q$. be a prime power. Now Mann [11], pp. 118-119, has shown that if $x$ is a primitive element of $G S(q)$, where $q=4 t+1$, then there exist odd integers $\alpha$ and $\beta$ such that

$$
\frac{x^{\alpha}+1}{x^{\alpha}-1}=x^{\beta} .
$$

Consider the set of $t$ blocks

$$
\left\{\left(1, x^{2 t}\right),\left(x^{\alpha}, x^{\alpha+2 t}\right)\right\} \oplus\left(1, x^{2}, x^{4}, \ldots ., x^{2 t-2}\right) .
$$

Now $x^{4 t}=1, x^{2 t}=-1$. Let $x^{2 t}-1=x^{s}$. Then the differences arising from the $2 t$ half-blocks in (5.2) are

$$
x^{s+2 i}, x^{s+2 i+\alpha}, i=0,2, \ldots 2 t-2 .
$$

Since $\alpha$ is odd the differences are symmetrically repeated once. Hence the $2 t$ half-blocks in (5.2) on development give the BIB design.

$$
v_{0}=4 t+1, b_{0}=2 t(4 t+1), r_{0}=4 t, k_{0}=2, \lambda_{0}=1 .
$$


Again consider the differences arising from the $t$ blocks (5.2), disregarding the partitions into halfblocks. Then in addition to (5.3), we will have the differences arising from cross pairs, i.e., pairs whose members belong to different half-blocks. Let $x^{\alpha}-1$ $=x^{c}, x^{\alpha}+1=x^{d}$. Then these differences can be written as

$x^{c+2 i}, x^{c+2 t+2 i}, x^{d+2 i}, x^{d+2 t+2 i}, i=0,1, \ldots ., 2 t-2$.

If we remember that in virtue of (5.1), $c-d$ is odd we see that in (5.5) each nonzero element of $G F(q)$ occurs twice. Hence the differences from the blocks (5.2) are symmetrically repeated thrice. On developing these blocks we get a BIB design with parameters,

$v^{*}=4 t+1, b^{*}=t(4 t+1), r^{*}=4 t, k^{*}=4, \lambda^{*}=3$.

Hence if we develop the initial blocks (5.2), keeping the half-blocks intact we get a bridge tournament design belonging to the series (B).

In particular we get solutions for $v=5,9,13,17,25$, $29,37,41,49$. If $v \leqslant 50$, this leaves only the values 21,33 and 45 of the form $4 t+1$. These will be considered in section 11 .

ExAmple (1). Let $t=1$. Then the required design has parameters $v=5, \quad b=5, \quad r=4, \quad p=2$, $\lambda_{1}=1, \lambda_{2}=2$. A primitive element of $G F(5)$ is 2 , and

$$
\frac{2+1}{2-1}=3=2^{3} .
$$

Hence $\alpha=1, \beta=3$, and the initial block is

$$
\{(1,4),(2,3)\} \text {. }
$$

The complete design obtained by developing it has the blocks

$$
\{(1,4),(2,3)\},\{(2,5),(3,4)\},\{(3,1),(4,5)\},
$$$$
\{(4,2),(5,1)\},\{(5,3),(1,2)\} \text {. }
$$

ExAmple (2). Let $t=2$. Then the required design has parameters $v=9, b=18, \quad r=8, \quad p=2, \lambda_{1}=1$, $\lambda_{2}=2$. Let $x$ be a primitive element of $G F\left(3^{2}\right)$ with minimum equation $x^{2}+x+2=0$. Then

$$
\frac{x+1}{x-1}=x,
$$

whence $\alpha=1, \beta=1$. The design is obtained by developing the initial blocks

$$
\left\{\left(1, x^{4}\right),\left(x, x^{5}\right)\right\},\left\{\left(x^{2}, x^{5}\right),\left(x^{3}, x^{7}\right)\right\} .
$$

ExAMPle (3). When $t=3$, the required design has the parameters $v=13, \quad b=39, \quad r=12, \quad p=2$, $\lambda_{1}=1, \lambda_{2}=2$. Now 2 is a primitive element of $G F$
(13) and

$$
\frac{2^{3}+1}{2^{3}-1}=2^{9}
$$

Hence we can take $\alpha=3, \beta=9$. The design is obtained by developing the initial blocks

$$
\{(1,12),(8,5)\},\{(4,9),(6,7)\},\{(3,10),(11,2)\} .
$$

\section{Designs Belonging to the Series (C), When $4 t+1$ is a Prime Power}

Let $4 t+1=q$ where $q$ is a prime power. We identify each element of $G F(q)$ with one object and take an additional object $\propto$. To construct the initial blocks for the required design, we shall use the solution for series (B) already obtained in section 5. Let $\alpha$ and $\beta$ be as in section 5. Let the initial block $\left\{\left(1, x^{2 t}\right)\right.$, $\left.\left(x^{\alpha}, x^{\alpha+2 t}\right)\right\}$ be replaced by two blocks

$$
\left\{\left(1, x^{2 t}\right),\left(\propto, x^{\alpha}\right)\right\},\left\{\left(x^{\alpha}, x^{\alpha+2 t}\right),(\propto, 1)\right\},
$$

and let the other initial blocks

$$
\left\{\left(1, x^{2 t}\right),\left(x^{\alpha}, x^{\alpha+2 t}\right)\right\} \oplus\left(x^{2}, x^{4}, \ldots, x^{2 t-2}\right),
$$

be retained as they are. Further let us repeat the whole set of initial blocks (5.2), i.e.,

$$
\left\{\left(1, x^{2 t}\right),\left(x^{\alpha}, x^{\alpha+2 t}\right)\right\} \oplus\left(1, x^{2}, x^{4}, \ldots, x^{2 t-2}\right) .
$$

We shall now use theorem (2), to show that the halfblocks in (6.1), (6.2), (6.3) when developed give the BIB design

$$
\begin{array}{r}
v_{0}=4 t+2, b_{0}=2(2 t+1)(4 t+1), r_{0}=2(4 t+1), \\
k_{0}=2, \lambda_{0}=2 .
\end{array}
$$

First note that apart from the two half-blocks $\left(\propto, x^{\alpha}\right),(\propto, 1)$ each half block of $(5.2)$ is included twice in (6.1), (6.2) and (6.3) taken together. Hence from what has been proved in section 5 , the differences arising from the half-blocks in (6.1), (6.2), (6.3), (after deletion of $\propto$ ) are symmetrically repeated twice. It is also easy to check that the condition (iii) of theorem 2 is also satisfied. This proves the required result.

We shall next use the same theorem to prove that the blocks in (6.1), (6.2), (6.3), (disregarding half-blocks) when developed lead to the BIB design

$$
\begin{aligned}
v^{*}=4 t+2 . b^{*}=(2 t+1)(4 t+1), \\
r^{*}=2(4 t+1), k^{*}=4, \lambda^{*}=6
\end{aligned}
$$

Comparing with the blocks (5.2), and disregarding $\propto$, we see that every pair of objects in (5.2), is repeated in $(6.2),(6.3)$ taken together except that the pair $x^{2 t}$, 
$x^{\alpha+2 t}$ in the first case is replaced by the pair $1, x^{\alpha}$ in the second case. Since $x^{2 t}=-1$, the differences $\pm x^{2 t}\left(x^{\alpha}-1\right)$ arising from $x^{2 t}, x^{\alpha+2 t}$ are the same as the differences $\pm\left(x^{\alpha}-1\right)$ arising from $\left(x^{\alpha}, 1\right)$. Also the pairs in (6.3) are the same as in (5.2). Hence it follows as in section 5 that the differences arising from the blocks obtained from (6.2), (6.3), (6.4), after the deletion of $\propto$, are symmetrically repeated six times. The condition (iii) of Theorem 2 is also easily checked. This proves the statement made above.

Taking together the two results proved we see that when the initial blocks (6.2), (6.3), (6.4) are developed leaving the half-blocks intact, and $\propto$ unchanged, we get a tournament design belonging to the series (C).

In particular we get the solutions for $u=6,10,14$, $18,26,30,38,42,50$. If $v \leqslant 50$, this leaves only the values 22,34 and 46 of the form $4 t+2$. These will be considered in section 12 .

EXAMPLE 1. Let $t=1$, then the required design has parameters $v=6, b=15, \quad r=10, p=2, \quad \lambda_{1}=2$, $\lambda_{2}=4$. The initial blocks obtained by using Example 1 section 5 are

$$
\{(1,4),(\propto, 2)\},\{(2,3),(\propto, 1)\},\{(1,4),(2,3)\} .
$$

EXAMPLE 2. Let $t=2$, then the required design has parameters $v=10, b=45, r=18, p=2, \lambda_{1}=2$, $\lambda_{2}=4$. The initial blocks obtained by using Example 2 section 5 are

$$
\left\{\left(1, x^{4}\right),(\propto, x)\right\},\left\{\left(x, x^{5}\right),(\propto, 1)\right\},\left\{\left(1, x^{4}\right),\left(x, x^{5}\right)\right\},
$$

and the block

$$
\left\{\left(x^{2}, x^{5}\right),\left(x^{3}, x^{7}\right)\right\}
$$

repeated twice. Here $x$ is an element of $G F\left(3^{2}\right)$, with the minimum $x^{2}+x+2=0$.

EXAMPLE 3. Let $t=2$. The initial blocks of the design with parameters $v=14, b=91, r=26, p=2$, $\lambda_{1}=2, \lambda_{2}=4$, are

$$
\{(1,12),(\propto, 8)\},\{(8,5),(\propto, 1)\},\{(1,12),(8,5)\},
$$

and the blocks

$$
\{(4,9),(6,7)\},\{(3,10),(11,2)\}
$$

each taken twice.

\section{The Method of Composition for Con- structing Tournament Designs}

Bose and Shrikhande [5] developed the method of composition for constructing BIB designs. This can be adapted for constructing tournament designs. The method depends on the use of pairwise balanced designs of index unity which were first introduced by Bose and Shrikhande [4] as auxiliary designs useful for the construction of mutually orthogonal Latin squares of nonprime power orders, especially $4 t+2$.
An arrangement of $v$ objects in $b$ sets is called a pairwise balanced design of index $g$ and type $\left(v ; k_{1}\right.$, $\left.k_{2}, \ldots, k_{m}\right)$ if each block contains either $k_{1}, k_{2} \ldots$ or $k_{m}$ distinct objects which are all distinct $\left(k_{i} \leqq v\right.$, $k_{i} \neq k_{j}$ ), and every pair of distinct objects occurs in exactly $g$ blocks of the design.

Now suppose there exists a pairwise balanced design $D$ of index $g$ and type $\left(v^{*} ; k_{1}^{*}, k_{2}^{*}, \ldots k_{m}^{*}\right)$, with $b^{*}$ blocks. Let the number of blocks with exactly $k_{i}^{*}$ treatments be $b_{i}^{*}$. Then clearly

$$
b^{*}=\sum_{i=1}^{m} b_{i}^{*}, g v(v-1)=\sum_{i=1}^{m} b_{i}^{*} k_{i}^{*}\left(k_{i}^{*}-1\right) .
$$

Also suppose there exist $m$ tournament designs $T_{i}$ with parameters

$v_{i}=k_{i}, b_{i}, r_{i}=n\left(v_{i}-1\right), p_{i}=p, \lambda_{1 i}=n(p-1), \lambda_{2 i}=n p$,

$(i=1,2, \ldots, m)$, then we can construct a tournament design $T$ with parameters

$$
\begin{aligned}
v=v^{*}, b=\sum_{i=1}^{m} b_{i} b_{i}^{*}, r & =g n(v-1), \\
& p, \lambda_{1}=g n(p-1), \lambda_{2}=g n p .
\end{aligned}
$$

The objects of the design $T$ will be the same as the objects of $D$. Let $B$ be a block of $D$ containing $k_{i}$ objects. We now obtain $b_{i}$ blocks of $T$ by writing down the $b_{i}$ blocks of design $T_{i}$, the objects being the objects occurring in $B$. Then among the $b_{i}$ blocks of $T$ which correspond to $B$, every pair of objects occurring together in $B$, will occur in $g \lambda_{1 i}$ half-blocks and $g\left(\lambda_{1 i}+\lambda_{2 i}\right)$ blocks. We now write down the $b_{i}$ blocks of $T$ corresponding to each of the $b_{i}^{*}$ blocks of $D$ which are of size $k_{i}$, obtaining altogether $b_{i} b_{i}^{*}$ blocks. Repeat-

ing this for $i=1,2, \ldots, m$ we get altogether $\sum_{i=1}^{m} b_{i} b_{i}^{*}$

blocks, in which every pair of objects from $D$, occurs $g n p$ times in half-blocks and $g n(2 p-1)$ times in blocks. We thus get the tournament design with parameters (7.3). The case $g=1$ is the most useful.

\section{Designs Belonging to the Series (D), When $t \equiv 1($ Mod 3)}

Consider the series (D) of bridge tournament designs, when $t=3 u+1$. The parameters become

$$
\begin{aligned}
v=12 u+4, b & =3(3 u+1)(4 u+1), \\
r & =3(4 u+1), p=2, \lambda_{1}=1, \lambda_{2}=2 .
\end{aligned}
$$

In particular the design $S_{4}$ with $u=0$ exists if $a, b, c$, $d$ be the objects. Then the design has the parameters

$$
v=4, b=3, r=3, p=2, \lambda_{1}=1, \lambda_{2}=2,
$$


and the blocks are

$$
\{(a, b),(c, d)\},\{(a, c),(b, d)\},\{(a, d),(b, c)\} .
$$

Now consider the BIB design with parameters

$$
\begin{aligned}
v^{*}=12 u+4, b^{*}= & (3 u+1)(4 u+1), \\
& r^{*}=(4 u+1), k^{*}=4, \lambda^{*}=1 .
\end{aligned}
$$

Note that a BIB design with $\lambda^{*}=1$ is a special type pairwise balanced design of index unity, where the blocks are all of one type. Taking into account Mann's Lemma given in section 5 , a solution to (8.4) can always be found by the method given by Bose in (1), when $4 u+1$ is a prime power. Hanani [8] has shown that a solution exists for all values of $u$.

If we use the method of composition taking (8.4) as $D$, and (8.2) as $T_{1}$, we get the solution for (8.1).

EXAMPLE 1. Let $u=1$. The required design has the parameters $v=16, b=60, r=15, p=2, \lambda_{1}=1$, $\lambda_{2}=2$. The corresponding $D$ has initial blocks (cf. [1])

$\left(1_{1}, 4_{1}, 2_{2}, 3_{2}\right),\left(1_{2}, 4_{2}, 2_{3}, 3_{3}\right),\left(1_{3}, 4_{3}, 2_{1}, 3_{1}\right),\left(\propto, 0_{1}, 0_{2}, 0_{3}\right)$,

which have to be developed $(\bmod 5)$, keeping $\propto$ and the subscripts invariant. Each initial block gives three initial blocks for the required design. Thus the initial blocks corresponding to $\left(1_{1}, 4_{1}, 2_{2}, 3_{2}\right)$ are

$\left\{\left(1_{1}, 4_{1}\right),\left(2_{2}, 3_{2}\right)\right\},\left\{\left(1_{1}, 2_{2}\right),\left(4_{1}, 3_{2}\right)\right\},\left\{\left(1_{1}, 3_{2}\right),\left(4_{1}, 2_{2}\right)\right\}$.

In the same way using solutions for (8.4) given in (1), we get the bridge tournament designs belonging to the series (D) for $v=28$ and 40.

\section{Designs Belonging to the Series When $t \equiv 0(\operatorname{Mod} 5)$ or $1(\operatorname{Mod} 5)$}

(D),

A solution for the bridge tournament design with parameters

$$
v=5, b=5, r=4, p=2, \lambda_{1}=1, \lambda_{2}=4,
$$

has already been given in Example 1, section 5. Now suppose a solution for the BIB design

$v_{0}=4 t+1, b_{0}=\frac{t(4 t+1)}{5}, r_{0}=t, k_{0}=5, \lambda_{0}=1$,

is known. If we delete one particular treatment from all the blocks we get a pairwise balanced design $D$ of type $(4 t ; 4,5)$ with $t$ blocks of size 4 , and $4 t(t-1) / 5$ blocks of size 5 . Now we use the method of composition taking for $T_{1}$ the design (8.2) and for $\left(T_{2}\right)$ the design (9.1). Then we obtain a solution for the bridge tournament design

$$
v=4 t, b=t(4 t-1), r=4 t-1, p=2, \lambda_{1}=1, \lambda_{2}=2 .
$$

Hence using known solutions from (1), for the BIB designs
(1) $v_{0}=21, b_{0}=21, r_{0}=5, k_{0}=5, \lambda_{0}=1$,
(ii) $v_{0}=41, b_{0}=82, r_{0}=10, k_{0}=5, \lambda_{0}=1$,
(iii) $v_{0}=25, b_{0}=30, r_{0}=6, k_{0}=5, \lambda_{0}=1$,
(iv) $v_{0}=45, b_{0}=99, r_{0}=11, k_{0}=5, \lambda_{0}=1$,

we get solutions for bridge tournament designs of the series (D) when $v=20,40,24,44$. Hanani has shown $[8,9]$ that a solution for $(9.2)$ always exists when $t \equiv 0(\bmod 5)$ or $1(\bmod 5)$. Hence under the same conditions, a solution for a bridge tournament design belonging to series (D) always exists.

\section{Some Pairwise Balanced Designs of Index Unity Obtained by Adjunction}

We have already seen how the method of composition enabled us to obtain solutions of bridge tournament designs with the help of suitable pairwise balanced designs of index unity. Here we shall describe a very useful method of deriving such designs, which will in the sequel be used for the construction of bridge tournament designs.

A BIB design is said to be resolvable if the blocks can be grouped into sets (called replications) such that each object occurs exactly once in each replication. Suppose-we have a resolvable BIB design with parameters $v^{*}, b^{*}, r^{*}, k^{*}, \lambda^{*}$. Let us take $c$ new treatments $\theta_{1}, \theta_{2}, \ldots, \theta_{c}(1 \leqslant c \leqslant r)$. To each block of the $i$ th replication add the treatment $\theta_{i}$, and in case $c>1$, add a new block $\left(\theta_{1}, \theta_{2}, \ldots, \theta_{c}\right)$. We then get a pairwise balanced design of index unity with $v$ $=v^{*}+c$ objects. Several cases have to be considered separately.

(a) If $c=1$, the new design obtained is of the type $\left(v^{*}+1 ; k^{*}+1, k^{*}\right)$, with $v^{*} / k^{*}$ of size $k^{*}+1$ and $b^{*}-\left(v^{*} / k^{*}\right)$ blocks of size $k^{*}$.

(b) If $c>1$ and $c \neq k^{*}, k^{*}+1$ or $r^{*}$ then the new design is of the type $\left(v^{*}+\mathrm{c} ; k^{*}+1, k^{*}, c\right)$ with $c v^{*} / k^{*}$ blocks of size $k^{*}+1, b^{*}-\left(c v^{*} / k^{*}\right)$ blocks of size $k^{*}$ and one block of size $c$.

(c) If $c=k^{*}<r^{*}$ then the new design is of the type $\left(v^{*}+c ; k^{*}+1, k^{*}\right)$ with $v^{*}$ blocks of size $k^{*}+1$ and $b^{*}-v^{*}+1$ blocks of size $k^{*}$.

(d) If $c=k^{*}+1<r^{*}$, then the new design is of the type $\left(v^{*}+c, k^{*}+1, k^{*}\right)$ with $1+\left\{\left(k^{*}+1\right) v^{*} / k^{*}\right\}$ blocks of size $k+1$ and $b-\left\{\left(k^{*}+1\right) v^{*} / k^{*}\right\}$ blocks of size $k^{*}$.

(e) If $c=r^{*}>k^{*}+1$ then the new design is of the type $\left(v^{*}+c, k^{*}+1, c\right)$ with $b^{*}$ blocks of size $k^{*}+1$ and one block of size $c=r^{*}$.

Three resolvable BIB designs which we shall use in this manner together with their solutions are given below [see Bose, Shrikhande, and Bhattacharya [6], table III, p. 171]. 


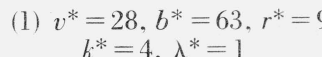

(2) $v=40, b=130, r=13$ $k=4, \lambda=1$
(3) $v=15, b=35, r=1$ $k=3, \lambda=1$
One replication is given by

$\left(01_{1}, 02_{1}, 10_{2}, 20_{2}\right),\left(21_{1}, 12_{1}, 22_{2}, 11_{2}\right)$

$\left(01_{2}, 02_{2}, 10_{3}, 20_{3}\right),\left(21_{2}, 12_{2}, 22_{3}, 11_{3}\right)$

$\left(01_{3}, 02_{3}, 10_{1}, 20_{1}\right),\left(21_{3}, 12_{3}, 22_{1}, 11_{1}\right)$ $\left(00_{1}, 00_{2}, 00_{3}, \propto\right)$.

Other replications are obtained by developing $(\bmod 3,3)$.

One replication is given by

$\left(1_{1}, 12_{1}, 8_{2}, 5_{2}\right),\left(4_{1}, 9_{1}, 6_{2}, 7_{2}\right)$,

$\left(3_{1}, 10_{1}, 11_{2}, 2_{2}\right)$

$\left(1_{2}, 12_{2}, 8_{3}, 5_{3}\right),\left(4.2,9_{2}, 6_{3}, 7_{3}\right)$,

$\left(3 ., 10,2,11_{3}, 22_{3}\right)$

$\left(1_{3}, 12_{3}, 8_{1}, 5_{1}\right),\left(4_{3}, 9_{3}, 6_{1}, 7_{1}\right)$,

$\left(3_{3}, 10_{3}, 11_{1}, 2_{1}\right)$

$\left(\propto, 0_{1}, 0_{2}, 0_{3}\right)$.

The other replications are obtained by developing $(\bmod 13)$

One replication is given by

$\left(1_{1}, 2_{1}, 4_{1}\right),\left(3_{1}, 1_{2}, 5_{2}\right),\left(6_{1}, 2_{2}, 3_{2}\right)$ $\left(5_{1}, 4_{2}, 6_{2}\right),\left(0_{1}, 0_{2}, \propto\right)$.

The other replications are obtained by developing $(\bmod 7)$.

\section{Designs Belonging to the Series for $t=5,8$ and 11}

(B),

When $t=5,8$ or 11 then $4 t+1$ is not a prime power. Hence the method of section 4 fails. A solution for these cases can however be obtained by using the method of composition.

(a) Let $t=5$. The required design has the parameters

$$
v=21, b=105, r=20, \lambda_{1}=1, \lambda_{2}=2 .
$$

The solution follows from the method of composition by using for $D$ the BIB design

$$
v^{*}=21, b^{*}=21, r^{*}=5, k^{*}=5, \lambda^{*}=1,
$$

and for $T_{1}$ the design $S_{5}$ with parameters (9.1) with the solution given in section 5, Example 1 . The solution for (11.2) is found by developing $\bmod 21$, the initial block [cf. [6], p. 170]

$$
(0,1,4,14,16) \text {. }
$$

(b) Let $t=8$. The required design has the parameters (10.3)

$$
v=33, b=264, r=32, p=2, \lambda_{1}=1, \lambda_{2}=2 .
$$

We start from a resolvable solution of the BIB design

$$
v^{*}=28, b^{*}=63, r^{*}=9, k^{*}=4, \lambda^{*}=1,
$$

given in section 10. By adjoining 5 new objects we get a pairwise balanced design $D$ of index unity and type $(33 ; 5,4)$ with 36 blocks of size 5 and 28 blocks of size 4. The required design is now obtained by applying the method of composition, using $D$ and taking for $T_{1}$ and $T_{2}$ the designs $S_{5}$ and $S_{4}$ with parameters (9.1) and (8.2).

(c) Let $t=11$. The required design has parameters

$$
v=45, b=495, r=44, p=2, \lambda_{1}=1, \lambda_{2}=2 \text {. }
$$

A solution to this can be obtained from the method of composition by using for $D$ the BIB design

$$
v^{*}=45, b^{*}=99, r^{*}=11, k^{*}=5, \lambda^{*}=1,
$$

and for $T_{1}$ the design $S_{5}$ with parameters (9.1). For a solution to $D$ see [1], p. 389 .

\section{Designs Belonging to the Series (C), for $t=5,8$, and 11}

Since $4 t+1$ is not a prime power when $t=5,8$ or 11, the method of section 6 fails. We can however use the method of composition to obtain the corresponding designs.

(a) Let $t=5$. The required design has the parameters

$$
v=22, b=231, r=42, p=2, \lambda_{1}=2, \lambda_{2}=4 .
$$

We start from a resolvable solution of the BIB design

$$
v^{*}=15, b^{*}=35, r^{*}=7, k^{*}=4, \lambda^{*}=1
$$

given in section 10. By adjoining 7 new objects we get a pairwise balanced design of index unity and type $(22 ; 4,7)$.

Let $S_{4}^{*}$ and $S_{7}^{*}$ be bridge tournament designs with parameters given by (12.3) and (12.4):

$$
\begin{array}{r}
v=4, b=6, r=6, p=2, \lambda_{1}=2, \lambda_{2}=4, \\
v=7, b_{2}=21, r_{2}=12, p=2, \lambda_{1}=2, \lambda_{2}=4 .
\end{array}
$$

Note that $S_{4}^{*}$ is not a minimal design. The solution for $S_{4}^{*}$ is simply obtained by duplicating the blocks (8.3). The solution for $S_{7}^{*}$ is given in section 4 , Example 1.

The required design (12.1) is now obtained by applying the method of composition using $D$ and taking $S_{4}^{*}$ and $S_{7}^{*}$ for $T_{1}$ and $T_{2}$.

(b) Let $t=8$. The required design has the parameters

$$
v=34, b=561, r=66, p=2, \lambda_{1}=2, \lambda_{2}=4 .
$$

We start from the resolvable solution of the BIB design

$$
v^{*}=28, b^{*}=63, r^{*}=9, k^{*}=4, \lambda^{*}=1,
$$


given in section 10 . By adjoining six new objects we get a pairwise balanced design $D$ of index unity and type $(34 ; 4,5,6)$ with 21 blocks of size 4,42 blocks of size 5 and one block of size 6 .

Let $S_{6}^{*}$ be the bridge tournament design with parameters

$$
v=6, b=15, r=10, p=2, \lambda_{1}=2, \lambda_{2}=4
$$

the solution for which is given in section 6, example (1). Also let $S_{5}^{*}$ be the design with parameters

$$
v=5, b=10, r=8, p=2, \lambda_{1}=2, \lambda_{2}=8
$$

the solution for which is obtained by duplicating the solution for (9.1).

The required solution for (12.5) not follows by applying the method of composition using $D$, and the designs $S_{4}^{*}, S_{5}^{*}$, and $S_{6}^{*}$ for $T_{1}, T_{2}$, and $T_{3}$. Note that the number of blocks obtained is

$$
21(6)+42(10)+1(15)=561
$$

which checks.

(c) Let $t=11$. The required design has the parameters

$$
v=46, \quad b=1035, \quad r=90, \quad p=2, \quad \lambda_{1}=2, \quad \lambda_{2}=4 .
$$

We start from the resolvable solution of the BIB design

$$
v^{*}=40, \quad b^{*}=130, \quad r^{*}=13, \quad k^{*}=4, \quad \lambda^{*}=1
$$

given in section 10 , and obtain from it a pairwise balanced design $D$ of index unity and type $(46 ; 4,5,6)$ with 70 blocks of size 4, 60 blocks of size 5 and one block of size 6 . Applying the method of composition using $D$, and the designs $S_{4}^{*}, S_{5}^{*}$, and $S_{6}^{*}$ as $T_{1}, T_{2}, T_{3}$ we get the required solution for (12.8).

\section{Designs Belonging to Series (D), for $t=2,3,8,9$, and 12}

(a) Let $t=2$. The required design has the parameters

$$
v=8, \quad b=14, \quad r=7, \quad p=2, \quad \lambda_{1}=1, \quad \lambda_{2}=2 .
$$

Let us associate one object to each element of $G F(7)$ and adjoin another object $\propto$. Then a solution to the required design is obtained by developing $\bmod 7$ the initial blocks

$$
\{(0,1),(4,2)\}, \quad\{(1,4),(2, \propto)\} .
$$

The proof follows by observing that from Theorem
(2), the two initial blocks in (13.2), disregarding halfblocks, provide a solution of the BIB design [see Bose [2], p. 30]

$$
v^{*}=8, \quad b^{*}=14, \quad r^{*}=7, \quad k^{*}=4, \quad \lambda^{*}=3,
$$

and the half-blocks provide a solution of the BIB design

$$
v^{*}=8, \quad b^{*}=28, \quad r^{*}=7, \quad k^{*}=2, \quad \lambda^{*}=1 .
$$

(b) Let $t=3$. The required design has the parameters

$$
v=12, \quad b=33, \quad r=11, \quad p=2, \quad \lambda_{1}=1, \quad \lambda_{2}=2 .
$$

Let us associate one object to each element of $G F(11)$, and adjoin another object $\propto$. Then a solution of the required design is obtained by developing $(\bmod 11)$ the initial blocks

$$
\{(0,1),(3,7)\}, \quad\{(4,9),(2,10)\}, \quad\{(\propto, 5),(6,8)\} .
$$

If we disregard the half-blocks, then the initial blocks (13.4) provide a solution of the BIB design

$$
v^{*}=12, \quad b^{*}=33, \quad r^{*}=11, \quad k^{*}=4, \quad \lambda^{*}=3 .
$$

This solution was given by Rao [12]. Also the halfblocks in (13.4) provide a solution of the BIB design

$$
v^{*}=12, \quad b^{*}=66, \quad r^{*}=22, \quad k^{*}=2, \quad \lambda^{*}=1 .
$$

(c) Let $t=8$ or 9 . The two required designs have the parameters

$$
\begin{aligned}
& v=32, \quad b=248, \quad r=31, \quad p=2, \quad \lambda_{1}=1, \quad \lambda_{2}=2 \\
& v=36, \quad b=315, \quad r=35, \quad p=2, \quad \lambda_{1}=1, \quad \lambda_{2}=2 .
\end{aligned}
$$

Starting from the resolvable solution of the BIB design with parameters

$$
v^{*}=28, b^{*}=63, r^{*}=9, k^{*}=4, \lambda^{*}=1,
$$

we can by adjoining $x$ treatments $(x=4,8)$, and a new block of size $x$ obtain pairwise balanced designs $D_{1}$ and $D_{2}$ of index unity and types $(32 ; 4,5)$ and $(36$; $4,5,8)$.

The solution of (13.7) follows by applying the method of composition using $D_{1}$ and the designs $S_{4}$ and $S_{5}$, with parameters (8.2) and (9.1), as $T_{1}$ and $T_{2}$.

Again let $S_{8}$ be the design with parameters (13.1). Then a solution of (13.8) follows by applying the method of composition using $D_{2}$ and the designs $S_{4}, S_{5}$, and $S_{8}$ for $T_{1}, T_{2}$, and $T_{3}$. 
(d) Let $t=12$. The required design has the parameters

$$
v=48, b=564, r=47, p=2, \lambda_{1}=1, \lambda_{2}=2 .
$$

We start from the resolvable solution of the BIB design

$$
v=40, b=130, r=13, k=4, \lambda=1,
$$

given in section 10 , and derive from it a pairwise balanced design $D$ of index unity and type $(48 ; 4,5,8)$, by adjoining 8 new treatments.

We can now apply the method of composition using $D$ together with the designs $S_{4}, S_{5}$, and $S_{8}$ as $T_{1}, T_{2}$, $T_{3}$ to obtain the solution of (13.9).

\section{Designs Belonging to the Series $(A)$, for $t=3,8$, and 9 and the Use of Group Divisible Designs}

(a) Let us first consider the case $t=8$. Then the required design has the parameters

$$
v=35, b=595, r=68, p=2, \lambda_{1}=2, \lambda_{2}=4 .
$$

We start from the resolvable solution of the BIB design

$$
v^{*}=28, b^{*}=63, r^{*}=9, k^{*}=4, \lambda^{*}=1,
$$

given in section 10 , and obtain from it a pairwise balanced design $D$ of index unity and type $(35 ; 4,5,7)$ with 14 blocks of size 4,49 blocks of size 5 , and one block of size 7 .

Let $S_{4}^{*}$ and $S_{5}^{*}$ be as in section 12 , and let $S_{7}^{*}$ be the bridge tournament design with parameters

$$
v=7, b=21, r=12, p=2, \lambda_{1}=2, \lambda_{2}=1,
$$

the solution for which is given in section 4, Example 1 .

The required solution for (14.1) is then obtained by applying the method of composition using $D$ together with the designs $S_{4}^{*}, S_{5}^{*}$ and $S_{7}^{*}$ for $T_{1}, T_{2}, T_{3}$. Note that the number of blocks obtained is

$$
14(6)+49(10)+21(1)=595 .
$$

(b) We have now obtained all minimal bridge tournament designs for $v \leqslant 50$, except the following two designs

$$
v=39, b=741, r=76, p=2, \lambda_{1}=2, \lambda_{2}=4,
$$

$$
v=15, b=105, r=28, p=2, \lambda_{1}=2, \lambda_{2}=4,
$$

both belonging to the series (A). We shall use group divisible (GD) designs for constructing the solution of these designs. For the combinatorial properties and the methods of construction for these designs refer to Bose and Connor [3] and Bose, Shrikhande, and Bhattacharya [6].

Given $v_{0}=m_{0} n_{0}$ objects divided into $m_{0}$ groups each with $n_{0}$ treatments, a GD design with parameters

$$
v_{0}, b_{0}, r_{0}, k_{0}, m_{0}, n_{0}, \lambda_{10}, \lambda_{20},
$$

is an arrangement of the objects in $b_{0}$ blocks each of size $k_{0}$, such that any two objects belonging to the same group occur together in $\lambda_{10}$ blocks, and any two objects belonging to different groups occur together in $\lambda_{20}$ blocks.

(b) For a solution of (14.3) we start from the GD design with parameters

$v_{0}=32, b_{0}=64, r_{0}=8, k_{0}=4$,

$$
m_{0}=4, n_{0}=8, \lambda_{10}=0, \lambda_{20}=1 \text {. }
$$

A method of obtaining a resolvable solution of this design is given in [6], table VII, p. 186. Let us take 7 new treatments $32+i(i=1,2, \ldots .7)$. To each block of the $i$ th replication add the treatment $32+i$. Also take as blocks the groups, and also add a new block consisting of the 7 new treatments. We then get a pairwise balanced design $D$ of index unity and type $(39 ; 4,5,7,8)$ with $8,56,1$ and 4 blocks of size $4,5,7$ and 8 respectively.

Let $S_{4}^{*}, S_{5}^{*}$, be as in section 12 , let $S_{7}^{*}$ be the design with parameters (14.2), and let $S_{8}^{*}$ be the design with parameters

$$
v=8, b=28, r=14, p=2, \lambda_{1}=1, \lambda_{2}=2,
$$

obtained by duplicating the design (13.1) whose solution is given in section 13(a).

We now get the required solution for (14.3) by applying the method of composition using (D), together with the designs $S_{4}^{*}, S_{5}^{*}, S_{7}^{*}$, and $S_{8}^{*}$ as $T_{1}, T_{2}, T_{3}, i_{4}$. Note that the number of blocks obiained is

$$
8(6)+56(10)+1(21)+4(28)=741 .
$$

(c) For a solution of (14.3) we have to start out from the GD design with parameters

$$
v=15, b=30, r=8, k=4, m=3, n=5, \lambda_{1}=1, \lambda_{2}=2
$$

[see Bose, Shrikhande, and Bhattacharya [6], table VI, No. (17), p. 178]. The treatments can be identified with pairs $(c, d)$ where $c$ belongs to $G F(5)$ and $d$ to $G F(3)$. Thus $\left(c_{1}, d_{1}\right)+\left(c_{2}, d_{2}\right)=(c, d)$ where $c_{1}+c_{2} \equiv c(\bmod 5), d_{1}+d_{9} \equiv d(\bmod 3)$. The three 


$$
\begin{array}{ll}
\text { Group I } & 00,10,20,30,40 ; \\
\text { Group II } & 01,11,21,31,41 ; \\
\text { Group III } & 02,12,22,32,42 .
\end{array}
$$

The blocks of (15.6) are obtained by developing the initial blocks

$$
(00,40,21,22), \quad(00,20,11,31,41), \quad(\bmod 5,3) \text {. }
$$

If to the 30 blocks constituting the solution of (15.6), we add the three groups as blocks, we get a pairwise balanced design $D$ of index 2 and type $(15 ; 4,5)$ with 30 blocks of size 4 and 3 blocks of size 5 . We now use the method of composition employing this design, and taking for $T_{1}$ and $T_{2}$ the designs $S_{4}$ and $S_{5}$ with parameters (8.2), (9.1). We then get the required solution for (14.3). Note that the number of blocks obtained is

$$
30(3)+3(5)=105 \text {. }
$$

(Paper 69B4-162)
[1] R. C. Bose, On the construction of balanced incomplete block designs, Ann. Eugen. 9, 353-399 (1939).

[2] R. C. Bose, On some new series of balanced incomplete block designs, Bull. Cal. Math. Soc. 34, 17-31 (1942).

[3] R. C. Bose and W. S. Conner, Combinatorial properties of group divisible incomplete block designs, Ann. Math. Stat. 23, 367-383 (1952).

[4] R. C. Bose and S. S. Shrikhande, On the construction of sets of mutually orthogonal Latin squares and the falsity of a conjecture of Euler, Trans. Amer. Math. Soc. 95, 191-209 (1960).

[5] R. C. Bose and S. S. Shrikhande, On the composition of balanced incomplete block designs, Can. J. Math. 177-188 (1960).

[6] R. C. Bose, S. S. Shrikhande, and K. N. Bhattacharya, On the construction of group divisible incomplete block designs, Ann. Math. Stat. 24, 167-195 (1953).

[7] Lewis Caroll, On the scheduling of lawn tennis tournaments, Complete works of Lewis Caroll, Modern Library.

[8] Haim Hanani, The existence and construction of balanced incomplete block designs, Ann. Math. 32, 361-386 (1961).

[9] Haim Hanani, A balanced incomplete block design, Ann. Math. Stat. 36, 711 (1965).

[10] M. Kraitchik, Mathematical Recreations (W. W. Norton and Co., Inc., New York, 1942).

[11] H. B. Mann, Analysis and Design of Experiments (Dover Publications, Inc., New York, 1949).

[12] C. R. Rao, A study of BIB designs with replications 11 to 15 , Sankhya, series A, 23, 117-127 (1961).

[13] Francis Scheid, A tournament problem, Amer. Math. Monthly 67, 39-41 (1960).

[14] C. C. Yalavigi, A tournament problem, Math. Student 31, 51-64 (1963). 